ESAIM: PROCEEDINGS AND SURVEYS, September 2014, Vol. 45, p. 456-466

J.-S. Dhersin, Editor

\title{
MODELING OF MOVING BOTTLENECKS IN TRAFFIC FLOW: A PDE-ODE MODEL WITH MOVING DENSITY CONSTRAINTS*
}

\author{
Maria Laura Delle Monache ${ }^{1}$
}

\begin{abstract}
In this article we study a strongly coupled PDE-ODE problem from an analytical and numerical point of view. This model describes a moving bottleneck that can be produced by the presence of a bus on a road network. The PDE is a scalar hyperbolic conservation law describing the traffic flow while the ODE, that describes the bus trajectory, is intended in Carathéodory sense. The moving constraint is given by an inequality on the flux which accounts for the bottleneck created by the bus on the road. We give an existence result and introduce a finite volume scheme. The algorithm uses a locally non-uniform moving mesh which tracks the bus position. Some numerical tests are presented, in order to describe the behavior of the solution.

Résumé. Dans cet article nous étudions un modèle couplé EDP-EDO d'un point de vue analytique et numérique et nous vérifions le caractère bien posé de ce problème. Le modèle a été introduit pour décrire un goulot d'étranglement mobile. En particulier, il peut décrire le mouvement d'un bus, qui roule à une vitesse inférieure à celle des autres voitures, réduisant la capacité de la route et générant un rétrécissement mobile de la chausée. L'EDP est une loi de conservation scalaire avec une contrainte mobile sur la densité et l'EDO décrive la trajectoire du bus. Nous présentons un résultat d'existence des solutions du modèle, obtenu par la méthode d'approximation de suivi des fronts, et nous montrons des simulations numériques obtenues avec une méthode "front-capturing".
\end{abstract}

\section{INTRODUCTION}

In the 1950s Lighthill and Whitham [17] and, independently, Richards [18] proposed the first macroscopic model for traffic known as the LWR model. They were the first ones to consider traffic flow as a fluid and to use fluid dynamics to describe its behavior on a single road. The traffic evolution is described with a non-linear scalar hyperbolic partial differential equation (PDE). In the last decade, several authors proposed different models to track the movement of a single car or a single vehicle among traffic, see $[2,5,8,16]$ and references therein. In all these works the dynamics of the single vehicle is described by an ordinary differential equation (ODE).

In this article, we focus on a strongly coupled PDE-ODE problem that was introduced in [13] to describe the effects of urban transport systems in a road network. The main traffic is described with a non-linear transport equation while the bus trajectory is described with an ODE and with an inequality constraint which describes the drop in the capacity of the road due to the presence of the slower vehicle, for example a bus. The solution of the ODE will be intended in Carathéodory sense. Compared to the previous models, the present one gives a more realistic description of the velocity of the slower vehicle and it is easier to handle both from the analytical

* This research was supported by the European Research Council under the European Union's Seventh Framework Program (FP/2007-2013) / ERC Grant Agreement n. 257661

${ }^{1}$ Inria Sophia Antipolis-Méditerranée; e-mail: maria-laura.delle_monache@inria.fr

(C) EDP Sciences, SMAI 2014 
and the numerical point of view.

This model can be considered a generalization of the model with fixed constraint studied in [1,6,7] to model toll gates and pedestrian flow. Subsequently, some numerical methods have been developed, see [9, 10, 12].

In this article, we give an existence result for the Cauchy problem with BV data and provide a finite volume algorithm which tracks the bus at each time step using a non-uniform moving mesh.

The article is developed as follows. Section 1 gives a description of the model from an analytical point of view. Section 2 is dedicated to the solution of the Riemann problem and Section 3 shows the existence of solutions for the Cauchy problem. Section 4 and 5 are dedicated to the numerical scheme and show some numerical tests.

\section{Mathematical MOdeL}

The goal of this work is to describe the phenomena caused by the presence of a bus in a car flow. Since the macroscopic description of the traffic does not allow to consider single vehicles, we consider the bus as a mobile obstacle that reduces the capacity of the road generating a moving bottleneck for the surrounding traffic. The traffic evolution is described by a scalar hyperbolic conservation law

$$
\partial_{t} \rho+\partial_{x} f(\rho)=0
$$

where the main quantities are the mean traffic density $\rho=\rho(t, x) \in[0, R]$ which is the scalar conserved quantity, with $R$ being the maximal density allowed on the road and the flux function $f:[0, R] \rightarrow \mathbb{R}^{+}$which is a strictly concave function such that $f(0)=f(R)=0$, see Figure 1a. It is given by the following flux-density relation

$$
f(\rho)=\rho v(\rho)
$$

where $v$ is a smooth decreasing function denoting the mean traffic speed and here set to be $v(\rho)=V\left(1-\frac{\rho}{R}\right)$, $V$ being the maximal velocity allowed on the road.

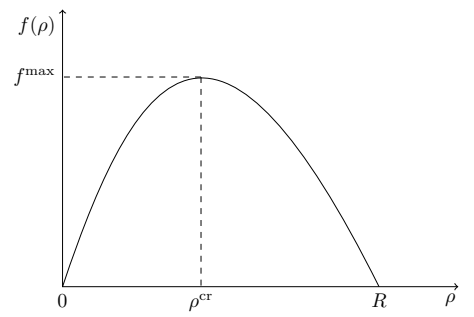

(A) One lane

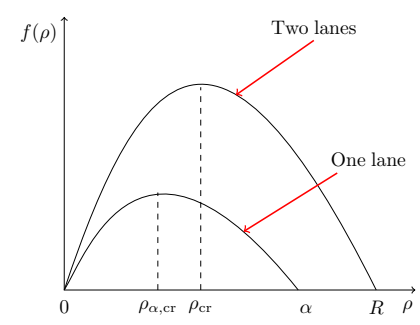

(B) Several lanes

FiguRE 1. Fundamental Diagram

Every road has a specific fundamental diagram. For example, for roads with different number of lanes we can consider as a first approximation that the fundamental diagrams are not affine and in a ratio that depends on the maximal capacities of the different lanes, see Figure 1b. The bus does not behave like cars hence, it cannot be modeled in the same way. We represent a single bus such that we can track its trajectory at all times. When it is possible, the bus will move at its own maximal speed which, we denote as $V_{b}<V$. When the surrounding traffic is too dense the bus will adapt its velocity to the one of the cars, so it will not be possible for the bus to overtake the cars. From a mathematical point of view, the velocity of the bus can be described by the following function:

$$
\omega(\rho)= \begin{cases}V_{b} & \text { if } \rho \leq \rho^{*} \doteq R\left(1-\frac{V_{b}}{V}\right) \\ v(\rho) & \text { otherwise }\end{cases}
$$


and the bus trajectory is described by the following ODE

$$
\dot{y}(t)=\omega(\rho(t, y(t)+))
$$

where $y$ denotes the position of the bus.

To describe the interaction between the bus and the traffic we consider the bus as a mobile obstacle, i.e., as a moving restriction of the road. The situation is the following: upstream and downstream with respect to the bus, the cars behave normally while on the side of the bus the road capacity is reduced, generating a bottleneck, see Figure 2.

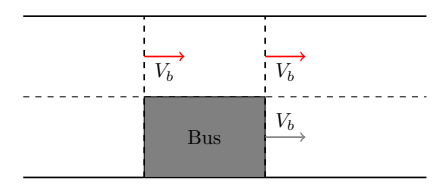

FiguRE 2. Moving bottleneck

This discontinuity moves at the bus speed. To better capture the influence of the bus, we choose to study the problem in the bus reference frame. This means setting $X=x-y(t)$. In this coordinate system the velocity of the bus is equal to zero. As a consequence the conservation law can be rewritten as:

$$
\partial_{t} \rho+\partial_{X}(f(\rho)-\dot{y} \rho)=0,
$$

with a corresponded a constraint on the flux that can be written in the following way

$$
f(\rho(t, y(t)))-\dot{y}(t) \rho(t, y(t)) \leq \frac{\alpha R}{4 V}(V-\dot{y}(t))^{2}
$$

with the constant coefficient $\alpha \in] 0,1[$ giving the reduced rate of the road capacity due to the presence of the bus. This constraint is obtained setting

$$
f(\rho(t, y(t)))-\dot{y} \rho(t, y(t)) \leq f_{\alpha}\left(\rho_{\alpha}\right)-\dot{y} \rho_{\alpha}
$$

where $f_{\alpha}(\rho)=\rho\left(1-\frac{\rho}{\alpha}\right)$ is the smaller fundamental diagram in Figure 3 and $\rho_{\alpha}=\frac{\alpha}{2}(1-\dot{y})$ the point that satisfies $f_{\alpha}^{\prime}\left(\rho_{\alpha}\right)=V_{b}$. This constraint is always satisfied if $\dot{y}(t)=v(\rho)$ since the left hand side is 0. Moreover, it is well defined because of the Rankine-Hugoniot conditions. This constraint describes mathematically the reduction of capacity of the road due to the presence of the bus.

For our purposes, it is not restrictive to assume that $R=V=1$, so that the full model writes

$$
\begin{cases}\partial_{t} \rho+\partial_{x}(\rho(1-\rho))=0, & (t, x) \in \mathbb{R}^{+} \times \mathbb{R}, \\ \rho(0, x)=\rho_{0}(x), & x \in \mathbb{R}, \\ f(\rho(t, y(t)))-\dot{y}(t) \rho(t, y(t)) \leq F_{\alpha} \doteq \frac{\alpha}{4}(1-\dot{y}(t))^{2}, & t \in \mathbb{R}^{+}, \\ \dot{y}(t)=\omega(\rho(t, y(t)+)), & t \in \mathbb{R}^{+}, \\ y(0)=y_{0} & \end{cases}
$$

The above model was introduced in [13], in an engineering framework, to study the effect of urban transport systems in a road network. Its analytical properties were addressed in [11]. Here we show some results but we refer the reader to [11] for a detailed explanation and proofs. 


\section{The Riemann PRoblem With MOVing DENSITy CONSTRAint}

Consider (7) with the particular choice of initital data

$$
y_{0}=0 \quad \text { and } \quad \rho_{0}(x)= \begin{cases}\rho_{L} & \text { if } x<0 \\ \rho_{R} & \text { if } x>0\end{cases}
$$

We are now ready to define the Riemann solver for $(7),(8)$ following $[13, \S \mathrm{V}]$. First, we recall the definition for a classical Riemann problem

Definition 2.1. The classical Riemann solver $\mathcal{R}$ for (1), with initial datum

$$
\rho_{0}(x)= \begin{cases}\rho_{L} & \text { if } x<0 \\ \rho_{R} & \text { if } x>0\end{cases}
$$

is the (right continuous) map $(t, x) \rightarrow \mathcal{R}\left(\rho_{L}, \rho_{R}\right)\left(\frac{x}{t}\right)$ that gives the standard weak entropy solution to (1). It is defined as follows. If $\rho_{L}<\rho_{R}$, then the entropy-admissible solution is given by the shock wave

$$
\rho(t, x)= \begin{cases}\rho_{L} & \text { if } x<\lambda t \\ \rho_{R} & \text { if } x>\lambda t .\end{cases}
$$

where, by the Rankine-Hugoniot condition we get $\lambda=\frac{f\left(\rho_{R}\right)-f\left(\rho_{L}\right)}{\rho_{R}-\rho_{L}}$. If, instead, $\rho_{L}>\rho_{R}$ the entropyadmissible solution to the Riemann problem is given by the rarefaction wave

$$
\rho(t, x)= \begin{cases}\rho_{L} & \text { if } x<f^{\prime}\left(\rho_{L}\right) t \\ \left(f^{\prime}\right)^{-1}\left(\frac{x}{t}\right) & \text { if } f^{\prime}\left(\rho_{L}\right) t<x<f^{\prime}\left(\rho_{R}\right) t \\ \rho_{R} & \text { if } x>f^{\prime}\left(\rho_{R}\right) t\end{cases}
$$
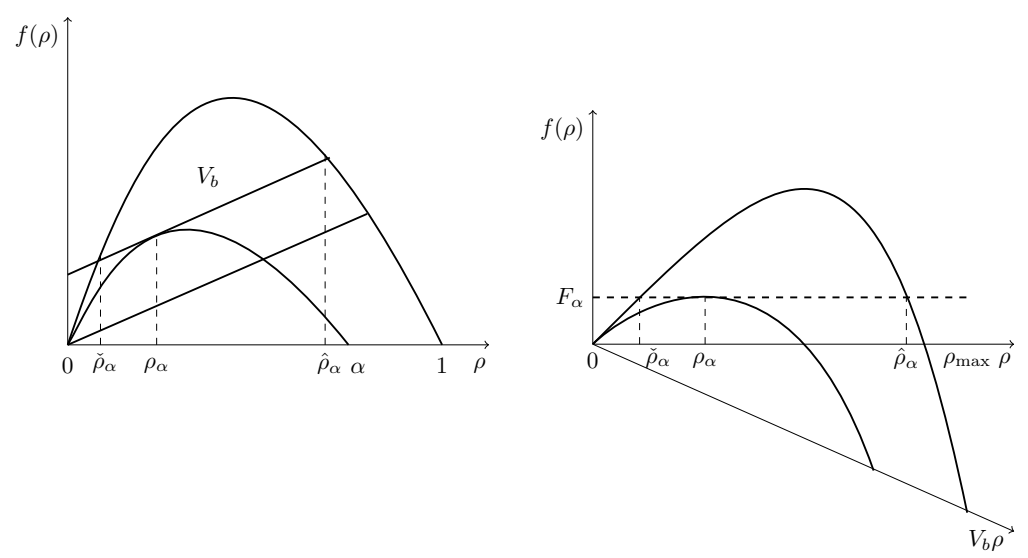

(A) Fixed reference frame

(B) Bus reference frame for $\dot{y}=V_{b}$

Figure 3. Flux functions. The big fundamental diagram describe the whole road and the smaller one the piece of road on the side of the bus.

Let $\check{\rho}_{\alpha}$ and $\hat{\rho}_{\alpha}$, with $\check{\rho}_{\alpha} \leq \hat{\rho}_{\alpha}$, be the two values of the density among which the flux constraint acts (see Figure 3 ) then, we can define the following. 
Definition 2.2. The constrained Riemann solver $\mathcal{R}^{\alpha}$ for (7), (8) is defined as follows.

(1) If $f\left(\mathcal{R}\left(\rho_{L}, \rho_{R}\right)\left(V_{b}\right)\right)>F_{\alpha}+V_{b} \mathcal{R}\left(\rho_{L}, \rho_{R}\right)\left(V_{b}\right)$, then

$$
\mathcal{R}^{\alpha}\left(\rho_{L}, \rho_{R}\right)(x)=\left\{\begin{array}{ll}
\mathcal{R}\left(\rho_{L}, \hat{\rho}_{\alpha}\right) & \text { if } x<V_{b} t, \\
\mathcal{R}\left(\check{\rho}_{\alpha}, \rho_{R}\right) & \text { if } x \geq V_{b} t,
\end{array} \quad \text { and } \quad y(t)=V_{b} t\right.
$$

(2) If $V_{b} \mathcal{R}\left(\rho_{L}, \rho_{R}\right)\left(V_{b}\right) \leq f\left(\mathcal{R}\left(\rho_{L}, \rho_{R}\right)\left(V_{b}\right)\right) \leq F_{\alpha}+V_{b} \mathcal{R}\left(\rho_{L}, \rho_{R}\right)\left(V_{b}\right)$, then

$$
\mathcal{R}^{\alpha}\left(\rho_{L}, \rho_{R}\right)=\mathcal{R}\left(\rho_{L}, \rho_{R}\right) \quad \text { and } \quad y(t)=V_{b} t .
$$

(3) If $f\left(\mathcal{R}\left(\rho_{L}, \rho_{R}\right)\left(V_{b}\right)\right)<V_{b} \mathcal{R}\left(\rho_{L}, \rho_{R}\right)\left(V_{b}\right)$, then

$$
\mathcal{R}^{\alpha}\left(\rho_{L}, \rho_{R}\right)=\mathcal{R}\left(\rho_{L}, \rho_{R}\right) \quad \text { and } \quad y(t)=v\left(\rho_{R}\right) t .
$$

Note that, when the constraint is enforced (point (1) in the Definition 2.2), a non-classical shock arises, which satisfies the Rankine-Hugoniot condition but violates the Lax entropy condition.

\section{The Cauchy problem: existence of solutions}

The aim of this section is to study the existence of the solutions of problem (7). We will prove existence of solutions of both the conservation law with the constraint and the ODE, at the same time.

As seen before, the bus influences the traffic along the road but it is also influenced by it. The bus position $y=y(t)$ solves

$$
\left\{\begin{array}{l}
\dot{y}(t)=\omega(\rho(t, y(t)+)) \\
y(0)=y_{0}
\end{array}\right.
$$

Solutions to (12) are to be intended in Carathéodory sense, i.e., as absolutely continuous functions which satisfy (12) for a.e. $t \geq 0$. We give our definition of solution.

Definition 3.1. A couple $(\rho, y) \in \mathrm{C}^{0}\left(\mathbb{R}^{+} ; \mathrm{L}^{1} \cap \mathrm{BV}(\mathbb{R} ;[0, R])\right) \times \mathrm{W}^{1,1}\left(\mathbb{R}^{+} ; \mathbb{R}\right)$ is a solution to $(7)$ if

(1) $\rho$ is a weak solution of the conservation law, i.e., for all $\varphi \in \mathrm{C}_{c}^{1}\left(\mathbb{R}^{2} ; \mathbb{R}\right)$

$$
\int_{\mathbb{R}^{+}} \int_{\mathbb{R}}\left(\rho \partial_{t} \varphi+f(\rho) \partial_{x} \varphi\right) d x d t+\int_{\mathbb{R}} \rho_{0}(x) \varphi(0, x) d x=0
$$

moreover, $\rho$ satisfies the Kružhkov entropy conditions [15] on $\left(\mathbb{R}^{+} \times \mathbb{R}\right) \backslash\left\{(t, y(t)): t \in \mathbb{R}^{+}\right\}$, i.e., for every $k \in[0,1]$ and for all $\varphi \in \mathrm{C}_{c}^{1}\left(\mathbb{R}^{2} ; \mathbb{R}^{+}\right)$and $\varphi(t, y(t))=0, t>0$,

$$
\int_{\mathbb{R}^{+}} \int_{\mathbb{R}}\left(|\rho-k| \partial_{t} \varphi+\operatorname{sgn}(\rho-k)(f(\rho)-f(k)) \partial_{x} \varphi\right) d x d t+\int_{\mathbb{R}}\left|\rho_{0}-k\right| \varphi(0, x) d x \geq 0 ;
$$

(2) $y$ is a Carathéodory solution of the ODE, i.e., for a.e. $t \in \mathbb{R}^{+}$

$$
y(t)=y_{0}+\int_{0}^{t} \omega(\rho(s, y(s)+)) d s
$$

(3) the constraint is satisfied, in the sense that for a.e. $t \in \mathbb{R}^{+}$

$$
\lim _{x \rightarrow y(t) \pm}(f(\rho)-\dot{y} \rho)(t, x) \leq F_{\alpha} .
$$

Remark that the above traces exist because $\rho(t, \cdot) \in \mathrm{BV}(\mathbb{R})$ for all $t \in \mathbb{R}^{+}$.

The following theorem ensures the existence of solutions. 
Theorem 3.2. Let $\rho_{0} \in B V(\mathbb{R} ;[0, R])$, then problem (7) admits a solution in the sense of Definition 3.1 .

In the rest of the section we give a sketch of the proof of Theorem 3.2. In particular, we will explain how to construct a sequence of approximate solutions via the wave-front tracking method, and prove its convergence. Finally, we will check that the limit functions satisfy conditions (13a)-(13d) of Definition 3.1. For the detailed proof we address the reader to [11].

\subsection{Wave-front tracking}

To construct piecewise constant approximate solutions, we adapt the standard wave-front tracking method, see for example $[3, \S 6]$. Fix a positive $n \in \mathbb{N}, n>0$ and introduce in $[0,1]$ the mesh $\mathcal{M}_{n}$ defined by $\mathcal{M}_{n}=$ $\left(2^{-n} \mathbb{N} \cap[0,1]\right) \cup\left\{\check{\rho}_{\alpha}, \hat{\rho}_{\alpha}\right\}$, modified in order to include the critical points $\check{\rho}_{\alpha}, \hat{\rho}_{\alpha}$. Moreover, let $f^{n}$ be the piecewise linear function that coincides with $f$ on $\mathcal{M}_{n}$, and let $\rho_{0}^{n}=\sum_{j} \rho_{0, j} \chi_{\left[x_{j-1}, x_{j}\right.}$ be a piecewise constant function with values in $\mathcal{M}_{n}$ which approximates $\rho_{0}$ in the sense of the strong $\mathrm{L}^{1}$ topology and $\operatorname{such} \operatorname{that} \mathrm{TV}\left(\rho_{0}^{n}\right) \leq \mathrm{TV}\left(\rho_{0}\right)$. We set $x_{0}=y_{0}$.

For small times $t>0$, a piecewise approximate solution $\left(\rho^{n}, y_{n}\right)$ to $(7)$ is constructed piecing together the solutions to the Riemann problems

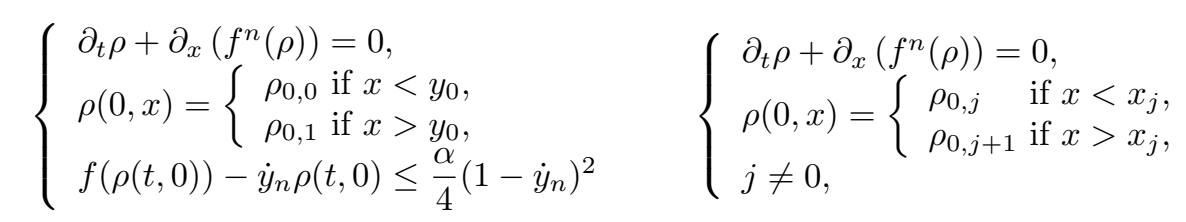

where $y_{n}$ satisfies

$$
\left\{\begin{array}{l}
\dot{y}_{n}(t)=\omega\left(\rho^{n}\left(t, y_{n}(t)+\right)\right) \\
y_{n}(0)=y_{0}
\end{array}\right.
$$

The approximate solution $\rho^{n}$ constructed above can be prolonged up to the first time $\bar{t}>0$, where two waves collide, or a discontinuity hits the bus trajectory. In both cases, a new Riemann problem arises and its solution, obtained in the former case with $\mathcal{R}$ and in the latter case with the constrained Riemann solver $\mathcal{R}^{\alpha}$, allows to extend $\rho^{n}$ further in time.

\subsection{Bounds on the total variation}

Due to the presence of non-classical shocks, the total variation may increase at interactions. To provide an uniform bound, we introduce the following Glimm-type functional. Given an approximate solution $\rho^{n}=\rho^{n}(t, \cdot)$ constructed by the wave-front tracking method, we define

$$
\Upsilon(t)=\Upsilon\left(\rho^{n}(t, \cdot)\right)=\operatorname{TV}\left(\rho^{n}\right)+\gamma=\sum_{j}\left|\rho_{j+1}^{n}-\rho_{j}^{n}\right|+\gamma
$$

where $\gamma$ is given by

$$
\gamma=\gamma(t)= \begin{cases}0 & \text { if } \rho^{n}\left(t, y_{n}(t)-\right)=\hat{\rho}_{\alpha}, \rho^{n}\left(t, y_{n}(t)+\right)=\check{\rho}_{\alpha} \\ 2\left|\hat{\rho}_{\alpha}-\check{\rho}_{\alpha}\right| & \text { otherwise. }\end{cases}
$$

The value of $\gamma$ is chosen to have the following uniform bound on $\Upsilon$.

Lemma 3.3. For any $n \in \mathbb{N}$, the map $t \mapsto \Upsilon(t)=\Upsilon\left(\rho^{n}(t, \cdot)\right)$ at any interaction either decreases by at least $2^{-n}$, or remains constant and the number of waves does not increase.

Lemma 3.3 in particular implies that the total number of waves is finite and therefore the wave-front tracking procedure can be prolonged to any time $T>0$. Moreover, it holds $T V\left(\rho^{n}(t)\right) \leq \gamma(t) \leq \gamma(0)=T V\left(\rho_{0}^{n}\right)+$ $2\left|\hat{\rho}_{\alpha}-\check{\rho}_{\alpha}\right|$. For the proof we refer the reader to [11]. 


\subsection{Convergence of approximate solutions}

In this section we prove that the limit of wave-front tracking approximations provides a solution $(\rho, y)$ of the PDE-ODE model (7) in the sense of Definition 3.1.

The following Lemma states the convergence of the wave-front tracking approximations.

Lemma 3.4. Let $\rho^{n}$ and $y_{n}, n \in \mathbb{N}$, be the wave-front tracking approximations to (7) constructed as detailed in Section 3.1, and assume $T V\left(\rho_{0}\right) \leq C$ be bounded, $0 \leq \rho_{0} \leq 1$. Then, up to a subsequence, we have the following convergences

$$
\begin{array}{lr}
\rho^{n} \rightarrow \rho & \text { in } \mathrm{L}_{\text {loc }}^{1}\left(\mathbb{R}^{+} \times \mathbb{R}\right) \\
y_{n}(\cdot) \rightarrow y(\cdot) & \text { in } \mathrm{L}^{\infty}([0, T]), \text { for all } T>0 ; \\
\dot{y}_{n}(\cdot) \rightarrow \dot{y}(\cdot) & \text { in } \mathrm{L}^{1}([0, T]), \text { for all } T>0
\end{array}
$$

for some $\rho \in \mathrm{C}^{0}\left(\mathbb{R}^{+} ; \mathrm{L}^{1} \cap B V(\mathbb{R})\right)$ and $y \in \mathrm{W}^{1,1}\left(\mathbb{R}^{+}\right)$.

Proof. (18a) is recovered using a standard procedure based on Helly's Theorem (see [3, Theorem 2.4]).

(18b) is given by an application of the Ascoli-Arzelà Theorem.

In order to prove $(18 \mathrm{c})$, we show that $\mathrm{TV}\left(\dot{y}_{n} ;[0, T]\right)$ is uniformly bounded.

For further details on this proof, we refer the reader to [11].

Proof of Theorem 3.2. Since $\rho^{n}$ converge strongly to $\rho$ in $\mathrm{L}_{\text {loc }}^{1}\left(\mathbb{R}^{+} \times \mathbb{R}\right)$, therefore it is straightforward to pass to the limit in the weak formulation of the conservation law, proving that the limit function $\rho$ satisfies (13a). Kružhkov entropy condition (13b) can be recovered in the same way.

To prove $13 \mathrm{c}$ we need to show that

$$
\lim _{n \rightarrow \infty} \rho^{n}\left(t, y_{n}(t)+\right)=\rho^{+}(t)=\rho(t, y(t)+) \quad \text { for a.e. } t \in \mathbb{R}^{+} .
$$

holds. At this purpose, we use the weak convergence of measures in [4, Lemma 15] and we proceed like in [4, §4], for the details see [11]. Combining (18c) and (19) we get $\dot{y}(t)=\omega(\rho(t, y(t)+))$ for a.e. $t>0$.

In order to verify that the limit solutions satisfy the constraint (13d), we can use directly the convergence result proved in the previous section.

\section{NuMERICAL SCHEME}

In this section we present a numerical method to compute solutions, including non-classical ones, to strongly coupled constrained PDE-ODE problems. We apply a front tracking capturing method which uses a Lagrangian algorithm in which the interface is tracked, such as the one in [19], together with a numerical method that tracks at each time step the slower vehicle trajectory, as in [5].

\subsection{Godunov-type scheme for hyperbolic PDEs with constraint}

We use the following notation: $x_{j+\frac{1}{2}}^{n}$ are the cell interfaces at time $t^{n}$ with $n \in \mathbb{N}$ and $j \in \mathbb{Z}$. A computational cell is given by $\left[x_{j-\frac{1}{2}}^{n}, x_{j+\frac{1}{2}}^{n}\right]$ where $x_{j}^{n}$ is the center of the cell and $h_{j}^{n}=x_{j-\frac{1}{2}}^{n}-x_{j+\frac{1}{2}}^{n}$ is the cell width at time $t^{n}$. Since we know the exact solutions of the Riemann Problem we develop a Godunov-type scheme. Classical Godunov scheme [14] can be expressed in conservative form as

$$
U_{j}^{n+1}=U_{j}^{n}-\frac{\Delta t}{\Delta x}\left(F\left(U_{j}^{n}, U_{j+1}^{n}\right)-F\left(U_{j-1}^{n}, U_{j}^{n}\right)\right)
$$

where $F(U, V)$ is the numerical flux. Boundary conditions are imposed on the left and on the right ends of the computational domain. Classical Godunov scheme does not represent all the characteristics of the solutions, 
failing to represent non-classical shocks.

Since our aim is to track the trajectory of the bus, and, also, represent non-classical solutions, a Lagrangian algorithm with a moving mesh is used. In particular, we develop an algorithm which follows at each time step the bus trajectory and modifies the mesh when the constraint enforces, that is when

$$
f\left(\mathcal{R}\left(\rho_{L}, \rho_{R}\right)\left(V_{b}\right)\right)>F_{\alpha}+V_{b} \mathcal{R}\left(\rho_{L}, \rho_{R}\right)\left(V_{b}\right)
$$

To keep non-classical shock on cell interfaces, we shift grid points locally and, as a consequence, we have a locally non-uniform mesh due to a cell interface moving with the bus trajectory. We use the superscript $n e w$ to indicate the quantities that are modified at time $t^{n}$ with the grid. Assume that at time $t^{n}, y^{n}$ is the bus position and $\left.y^{n} \in\right] x_{m-\frac{1}{2}}^{n}, x_{m+\frac{1}{2}}^{n}$ for some $m$. When the constraint (21) is enforced, the algorithm for the adaptive mesh reads as follows:

- If $\left|x_{m+\frac{1}{2}}^{n}-y^{n}\right|>\frac{h_{m}^{n}}{2}$ then move the point $x_{m-\frac{1}{2}}^{n}$ to $x_{m-\frac{1}{2}}^{n e w}=y^{n}$ and recompute the cell averages in the cells $m-1$ and $m$ from the formula

$$
U_{m-1}^{n e w}=\frac{\Delta x_{m-1}^{n} U_{m-1}^{n}+\left(x_{m-\frac{1}{2}}^{n e w}-x_{m-\frac{1}{2}}^{n}\right) U_{m}^{n}}{\Delta x_{m-1}^{n e w}}
$$

with $\Delta x_{m-1}^{n e w}=x_{m-\frac{1}{2}}^{n e w}-x_{m-\frac{3}{2}}^{n}$.

- If $\left|x_{m+\frac{1}{2}}^{n}-y^{n}\right| \leq \frac{h_{m}^{n}}{2}$ we adjust the location of the point $x_{m+\frac{1}{2}}^{n}$ such that $x_{m+\frac{1}{2}}^{n e w}=y^{n}$ and then we place the point $x_{m-\frac{1}{2}}^{n e w}$ at the middle distance between $x_{m-\frac{3}{2}}^{n}$ and $x_{m+\frac{1}{2}}^{n e w}$. We then compute the new cell averages in the cells $m$ and $m+1$ from the formulas

$$
\begin{aligned}
U_{m}^{n e w} & =\frac{\left(x_{m-\frac{1}{2}}^{n}-x_{m-\frac{1}{2}}^{n e w}\right) U_{m-1}^{n}+\left(x_{m+\frac{1}{2}}^{n e w}-x_{m+\frac{1}{2}}^{n}\right) U_{m}^{n}}{\Delta x_{m}^{n e w}} \\
U_{m+1}^{n e w} & =\frac{\Delta x^{n} U_{m+1}^{n}+\left(x_{m+\frac{1}{2}}^{n}-x_{m+\frac{1}{2}}^{n e w}\right) U_{m}^{n}}{\Delta x_{m+1}^{n e w}}
\end{aligned}
$$

with $\Delta x_{m}^{n e w}=x_{m+\frac{1}{2}}^{n e w}-x_{m-\frac{1}{2}}^{n e w}$ and $\Delta x_{m+1}^{n e w}=x_{m-\frac{1}{2}}^{n e w}-x_{m-\frac{3}{2}}^{n}$.

Each time the constraint is enforced the bus position follows the non-classical shock trajectory: $y^{n+1}=x_{m \pm \frac{1}{2}}^{n+1}=$ $x_{m \pm \frac{1}{2}}^{n e w}+V_{b} \Delta t^{n}$. The other cell interfaces are kept unchanged.

An explicit formula for the scheme can be derived and looks as follows

$$
\begin{aligned}
U_{j}^{n+1} & =\frac{\Delta x_{j}^{n}}{\Delta x_{j}^{n+1}} U_{j}^{n}-\frac{\Delta x_{j}^{n}}{\Delta x_{j}^{n+1}}\left[\left(\int_{t^{n}}^{t^{n+1}} f\left(\mathcal{R}\left(U_{j}^{n}, U_{j+1}^{n}\right)\right)-V_{b} \mathcal{R}\left(U_{j}^{n}, U_{j+1}^{n}\right)\right) d t\right. \\
& \left.-\left(\int_{t^{n}}^{t^{n+1}} f\left(\mathcal{R}\left(U_{j-1}^{n}, U_{j}^{n}\right)\right)-V_{b} \mathcal{R}\left(U_{j-1}^{n}, U_{j}^{n}\right)\right) d t\right] .
\end{aligned}
$$

For simplicity we introduce the notation $\tilde{F}(U)=F(U)-V_{b} U$. Notice that in our case $\tilde{F}$ corresponds to the $F_{\alpha}$ when the constraint is active and to $F(U)$ with $V_{b}=0$ when the constraint is not enforced. Moreover, one needs to be careful when the bus trajectory is dealt with. In fact, the cell size changes as time increases. In fact, after the mesh has been resized and adjusted, we update the cell averages for all cells with the following conservative formula:

$$
U_{j}^{n+1}=\frac{\Delta x_{j}^{n e w}}{\Delta x_{j}^{n+1}} U_{j}^{n}-\frac{\Delta t^{n}}{\Delta x_{j}^{n+1}}\left(\tilde{F}\left(U_{j}^{n}, U_{j+1}^{n}\right)-\tilde{F}\left(U_{j-1}^{n}, U_{j}^{n}\right)\right)
$$



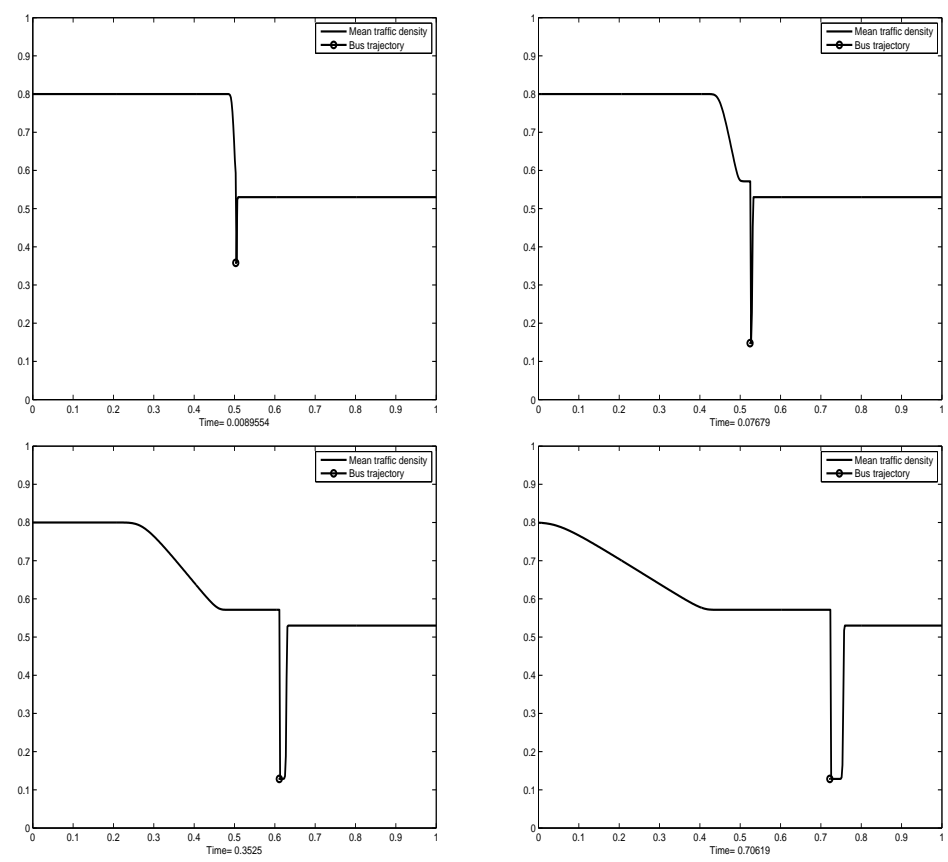

FIGURE 4. Evolution of the density at different times corresponding to initial data (27) and a mesh grid of 500 points.

\subsection{Numerical method for the ODE}

At each time $t^{n}$ we determine the position $y^{n}$ of the driver by studying interactions between the bus trajectory and the density waves within a fixed cell. We distinguish two cases:

- Constraint (21) is satisfied. Then the bus moves always at velocity $V_{b}$ and the bus position is updated $y^{n+1}=V_{b} \Delta t^{n}+y^{n}$.

- Constraint (21) is not satisfied. In this case we implement the tracking algorithm introduced in [5].

\section{Numerical ReSults}

In this section we present a numerical test performed with the scheme previously described. We display some numerical results obtained applying the modified Godunov scheme to problem (7). Here we deal with a road of length 1 parameterized by the interval $[0,1]$. In all the simulations we fix $V_{b}=0.3, \alpha=0.6$. We consider the following initial data

$$
\rho(0, x)=\left\{\begin{array}{ll}
0.8 & \text { if } x<0.5, \\
0.53 & \text { if } x>0.5,
\end{array} y_{0}=0.5 .\right.
$$

The values of the initial conditions create a rarefaction wave followed by a non-classical and a classical shocks on the density, as illustrated in Figure 4 and 5. Note the non-classical downward jump at the bus location.

\section{Conclusions}

This article introduces a strongly coupled PDE-ODE problem with a moving density constraint which can model the presence of a moving bottleneck on a road network. We proved the existence of solutions for general BV data for the Cauchy problem and proposed a Lagrangian finite volume scheme that is able to display non-classical solutions. Stability of the problem and a conservative numerical method are under study. 


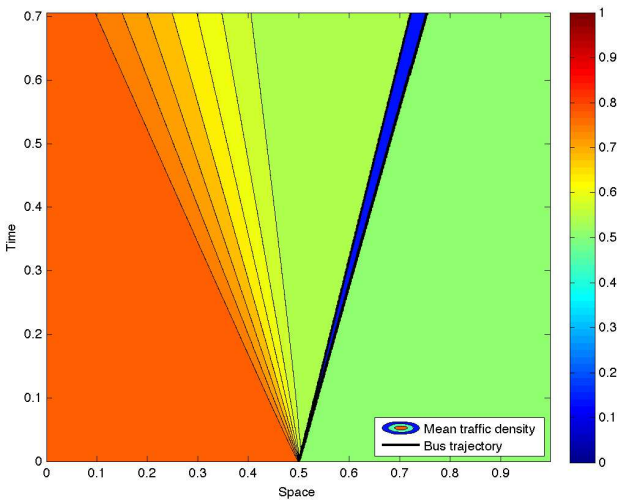

FIGURE 5. Density and bus trajectory in a $x-t$ plane corresponding to initial data (27) and a mesh grid of 500 points.

\section{REFERENCES}

[1] B. Andreianov, P. Goatin and N. Seguin, Finite volume scheme for locally constrained conservation laws, Numer. Math., 115 (2010), 609-645.

[2] R. Borsche, R. M. Colombo and M. Garavello, Mixed systems: ODEs - Balance laws, Journal of Differential equations, 252 (2012), 2311-2338.

[3] A. Bressan, "Hyperbolic systems of conservation laws - The one dimensional Cauchy problem," 1st edition, Oxford university press, 2000.

[4] A. Bressan and P. G. LeFloch, Structural stability and regularity of entropy solutions to hyperbolic systems of conservation laws, Indiana University Mathematical Journal, 48 (1999), 43-84.

[5] G. Bretti and B. Piccoli, A tracking algorithm for car paths on road networks, SIAM Journal of Applied Dynamical Systems, 7 (2008), 510-531.

[6] C. Chalons, P. Goatin and N. Seguin, General constrained conservation laws. Application to pedestrian flow modeling, Netw. Heterog. Media, 8(2) (2013), 433-463.

[7] R. M. Colombo and P. Goatin, A well posed conservation law with variable unilateral constraint, Journal of Differential Equations, 234 (2007), 654-675.

[8] R. M. Colombo and A. Marson, A Hölder continuous O.D.E. related to traffic flow, The Royal Society of Edinburgh Proceedings A, 133 (2003), 759-772.

[9] C.F. Daganzo and J.A. Laval, On the numerical treatement of moving bottlenecks, Transportation Research Part B 39 (2005), $31-46$.

[10] C.F. Daganzo and J.A. Laval, Moving bottlenecks: A numerical method that converges in flows, Transportation Research Part B 39 (2005), 855-863.

[11] M. L. Delle Monache and P. Goatin, Scalar conservation laws with moving density constraints, INRIA Research Report, n.8119 2012, http://hal.inria.fr/hal-00745671.

[12] M. L. Delle Monache and P. Goatin, A front tracking method for a strongly coupled PDE-ODE system with moving density constraints in traffic flow Discrete Contin. Dyn. Syst. Ser. S 7(3) (2014), 435-447.

[13] F. Giorgi, "Prise en compte des transports en commun de surface dans la modélisation macroscopique de l'écoulement du trafic," Ph.D thesis, Insitut National des Sciences Appliquèes de Lyon, 2002.

[14] S. K. Godunov, A finite difference method for the numerical computation of discontinuous solutions of the equations of fluid dynamics, Matematicheskii Sbornik, 47 (1959), 271-290.

[15] N. Kružhkov, First order quasilinear equations with several independent variables, Matematicheskii Sbornik, 81 (1970), 228255.

[16] C. Lattanzio, A. Maurizi and B. Piccoli, Moving bottlenecks in car traffic flow: a PDE-ODE coupled model, SIAM Journal of Mathematical Analysis, 43 (2011), 50-67.

[17] M. J. Lighthill and G. B. Whitham, On kinetic waves. II. Theory of traffic flows on long crowded roads, Proceedings of the Royal Society of London Series A, 229 (1955), 317-345.

[18] P. I. Richards, Shock waves on the highways, Operational Research, 4 (1956), 42-51. 
[19] X. Zhong, T. Y. Hou and P. G. LeFloch, Computational Methods for propagating phase boundaries, Journal of computational physics, 124 (1996), 192-216. 